\title{
Eric Charlebois
}

\section{Sempervirens}

Un poème pour redonner vie aux feuilles mortes.

Le syndrome de la plage blanche

de celle que tu es.

Ceindre aux muscles pectoraux le scapulaire que je serai toujours.

C'est l'automne ; il y a une odeur de cheminée qui n'a pas été ramonée, mêlée aux cendres du poêle extérieur qu'il faut serrer dans la fosse au loin.

C'est l'autre honneur, que de se gargariser des huées des écureuils qui s'approvisionnent en cartouches de pistolet à air comprimé.

Un abîme abîmé par les lézardes du vide dans notre visage inquiet.

Des nénuphars dans la piscine.

Des feuilles humides en guise de signet dans le guide de fabrication de l'amour, dénué du moindre mot.

Un gazebo comme un accent circonflexe sur le euh de l'incertitude.

Je trempe une feuille morte dans l'encre des meurtrissures ; nous nous sommes cognés si souvent sur les immeubles de nos corps.

Nota Malus : le mal de tête vient du cidre de la pomme défendue, qui ne tombe jamais très loin de nos mains, prêtées à SIMDUT pour la confection du symbole des matières corrosives. 
J'ai vu une chienne qui poussait son chiot dans un landau et qui tenait un enfant en laisse ; je me suis dit que l'humain est le meilleur ami du chien, et j'ai pensé à tout ce temps que l'on perd à lire les journaux jonchés de nouvelles déjà vieilles et jaunies par les soupirs d'impuissance.

C'est l'automne; la terre s'endort, le ciel s'éveille et le vent a une érection.

C'est l'automne : nos lèvres sont mouillées comme les feuilles, et les sapins n'ont pas hâte à Noël.

C'est l'automne, et le mélèze perd ses aiguilles et le buis préserve ses feuilles : c'est le monde à l'envers comme un vomissement de marbre sur un plancher de lasagne.

C'est l'automne, donc c'est dimanche tous les jours.

C'est l'automne, et mon chum s'est enlevé la vie comme on retire ses chaussures en entrant chez le monde à l'endroit.

C'est l'automne, et il y a une vente-débarras chez Dairy Queen.

C'est l'automne, et il y a de la crème glacée in vitro derrière les lunettes fumées.

C'est l'automne, et on fait des festivals pour se payer un foyer pour y brûler tout le bois mort voué à des feux de qu'en dira-t-on qui n'ont pas eu lieu.

C'est l'automne, et les retailles de cèdres pendent à l'échafaud.

C'est l'automne, et les dragons de Komodo de tes yeux lorgnent la charogne de mon corps que la voirie a oublié en bordure de ta route.

C'est l'automne, et les gens riches et chiches font leurs préparatifs pour le sud, dans la redoute d'un tout-inclus qui les protège de la misère qui, seule, a pitié d'eux.

C'est l'automne, et, après la pluie, la pluie.

C'est l'automne, et les mendiants injectent nos économies dans un cercueil de poésie.

C'est l'automne, et il y a comme un cerne autour du verre de Sangria, juste sous la constellation de la Table de Patio. 
Mon jumeau est autiste.

Son jumeau est un illuminé.

Je coupe le ruban inaugural du périmètre de sécurité du sourire de fumeur qui est dans le miroir mouroir.

Tu esquisses un sourire de verrou à chaîne à tenon et oui, et tu ouvres la porte cochère de ton jardin sous laquelle j'avais rampé à la chasse aux couleuvres sans colonne autour de la moelle à bois.

La génération Tim Hortons sans apostrophe : la dérouler sur le rebord pour faire des enfants en service au volant, et des coins à ordures et à recyclage comme des xylophones et des villes en plastique non recyclable. Un peu de café avec votre acide à batterie et votre quadroxyde de carbone?

Le drapeau devant le salon funéraire est toujours en berne ; il bat au vent des sanglots. Le temps est de l'argent terni dans les poches d'un numismate qui demande l'aumône devant le portail de la Monnaie royale canadienne.

C'est l'automne dans tes yeux vertueux, avertis, ouverts, vert Clorets.

On fait jouer un vieux vinyle sous l'aiguille d'un ouvre-boîte.

Grésillement, crépitement, chuintement, puis les premières guimauves de l'hiver qui cuisent dans les réacteurs d'un avion pendu au plafond, en guise de mobile, dans la chambre à couver, dans la chambre à air, dans la chambre de commerce.

L'écologie reste au centre de tous les débats : on vocifère, on vitupère, on vilipende, on carbonise, donc, entre deux cafés, vingt cigarettes et un océan d'eau embouteillée. L'effet de serre et laisser-faire.

C'est l'automne, et les poèmes poussaient dans les arbres et les poètes y pendent. C'est l'automne, et seules des feuilles d'aspenite tombent de tes yeux de réserve faunique. 
Tu clignes, et j'ai le droit de tourner à gauche.

Il y a un ralentisseur peint avec ton rimmel, sur ma route; j' accélère, parce que tout poème est une contravention.

Ligature des tromperies entre deux éléphants : notre tour d'ivoire est sans défense.

Je t'aime, et j'aime que tu m'aimes ; il reste qu'il faudrait remiser la toile du trampoline pour l'hiver; tous ses ressorts rouillés pendent comme des points d'interrogation.

Il nous a fallu nous habituer à garder un rouleau de papier hygiénique en réserve, par terre, à côté de la cuvette, au cas où une envie nous prenne d'écrire un parchemin de poésie.

Je dois produire un plan d'affaires : allons nous promener en mobile pour que je topographie ton corps, en surplomb.

Ton regard de Ginger Ale apaise la bile qui m'emplit comme un radeau de sauvetage dans une salle de musée.

C'est l'automne, et nous faisons nos provisions pour le grand ménage du printemps. 
Je demanderai un râteau pour Noël ; c'est sûr, qu'une élagueuse réglerait beaucoup de tracas, mais je pense à tout ce ciel hippopotame qui serait beaucoup trop accessible, sans l'ombre d'un boute.

Ton regard de lame de tondeuse me fauche l'air sous les pieds : je ne flotte bel et bien pas : j'ai les pieds dans l'embrasure de ton rire.

C'est l'automne, et nous n'avons pas vu d'oiseaux migrateurs

dans les cages

que transportait la remorque.

Il faut arrêter au poste d'inspection quand les feux clignotent ; je m'y soumets, pour me rendre compte que nous avons le poids permis ; notre passé nous a quittés.

C'est l'automne, et les jalons le long de l'autoroute sont tout à coup percutants.

C'est l'automne, et je t'attendrai devant l'entrée de la ruche.

C'est l'automne, et les moutons dans le pré transhumeront dans notre endormissement.

C'est l'automne, et c'est le tracas de le dire.

C'est l'automne, et les astronautes sont dans la lune, et les morts sont dans la terre.

C'est l'automne, n'ayons pas peur des motards.

C'est l'automne, et les enfants se tiennent par la main parce qu'ils ne peuvent se tenir trop par là.

C'est l'automne, et on dit qu'il faut laisser un linceul de feuilles dans les plates bandes dessinées de notre enfance. 
Tout est vert et Glad.

Tout est noué et flexible et imperméable et hydrocarburé comme nous dans la tente, au camping derrière l'église.

Nous croisons un stand à patates volé.

Nous nous disons que ça pourrait être pire : il aurait pu être plein d'écoliers.

Il y une aurore boréale derrière toi, sur la bande vidéo, comme il y a un dessin, là, derrière moi, sur le frigo ; on se demande alors si la réalité n'est pas plutôt dessinée que cinémascopique.

Tout est vertigineux quand nous nous couchons sur le dos d'une licorne.

C'est l'automne, et il y a un dénominateur commun à toutes ces fractions de seconde : les journées raccourcissent, et nos incarcérations charnelles se prolongent. C'est l'automne, et le brouillard a peur de digresser.

C'est l'automne, et j'ai envie de faire un feu de croix.

C'est l'automne, et nous nous frictionnons l'un à l'autre, mais aucune flammèche : nous ne sommes pas assez de pierre.

C'est l'automne, et ton regard de gélatine Advil me soulage de mon blues pâle.

C'est l'automne, et les nautoniers ont remisé leur chalutier dans nos glandes lacrymales, à l'abri du gel.

C'est l'automne, et je te refuse l'accès à mon laptop; tu es déjà sur mes cuisses.

C'est l'automne, et je suis content de vivre sans contentement, sans te tenir pour acquis, mais pour ce que tu es, toujours, insaisissable, comme une luciole pour les tuyaux des services d'incendie.

C'est l'automne, et je suis écœuré d'entendre tout le monde s'en servir comme prétexte pour expliquer la grisaille, le manque d'ardeur et la torpeur. 
Je t'ai apporté des fleurs des champs cueillies dans une plate-bande du voisinage.

Je t'ai offert du chocolat, une tablette d'Aero.

Je t'ai dit que tu étais belle, dans ma mémoire.

Je t'ai embrassé en cognant des dents.

Je m'étais aspergé d'eau de toilette publique.

Je suis arrivé à l'heure arrêtée.

Je t'ai sortie, pour souper, chez Tim Hortons.

Je t'ai regardée sans te toucher, comme dans une boutique de cristal, puis je me suis rendu compte que mes clés étaient mal placées, dans mes poches, et que j'avais vraiment besoin d'une tirelire.

Quand nous nous sommes réveillés, c'était l'automne, et l'ombre cherchait la marmotte. C'était l'automne, et les feuilles étaient mortes et enterrées, puisque tout ce que nous nous écrivions était transmis instantanément et électroniquement : la technologie est merveilleuse : nous n'étions plus jamais dans la peur de l'éloignement : nous étions dans le désir de combler une distance glauque. 
Glauque. Je répète le mot.

Glauque.

Comme l'eau d'une écluse.

Comme le dernier verre de rye laissé sur le bar.

Comme une gomme Trident Layers ${ }^{\mathrm{TM}}$ pomme et ananas.

Comme la Terre vue de la Lune de ton cou.

Comme mousse qui court n'amasse pas pierre.

Comme un pouding de rhubarbe.

Comme des amortisseurs de feutre taillés dans des chapeaux d'Oktoberfest.

Comme une feuille de papier à sableuse usé sur l'escalier de la véranda volé au littoral du ciel.

Comme du limon autour de nos doigts foreurs.

Comme l'opacité d'un feu rouge qui n'est pas passé au jaune.

Glauque comme une bouteille à la mer enroulée dans un phylactère de SOS. 
Le vent ternit, et le soleil se fait bronzer.

Naguère, nous regardions des photos de maintenant.

Nous consultons les données du dernier recensement pour établir le nombre d'invitations que nous posterons pour la consécration solennelle, silencieuse, obséquieuse, étanchement surveillée et hyper démocratique du nouveau couple royal : nous procéderons à une transfusion sanguine.

Je te parle d'un rôti de bœuf ; tu me parles d'une tentative de suicide.

Je te parle de rendez-vous et de rendons-nous; tu me parles de bulles teintes.

Je te parle des tâches d'ensevelissement qui nous incombent, avant la première neigerie ; tu me parles du scrapbooking auquel tu t'adonnes avec les chèques que tu ne déposes pas. Je te parle de la maison à rénover ; tu me parles des rénovations en quête de maisonnée. Je te parle de ramasser les feuilles ; tu me parles d'arroser la pelouse pour qu'en éclose une patinoire.

Je te parle de la qualité des sacs à ordures ; tu me parles des taxes municipales punies si mal.

Je te parle d'entreposage ; tu me parles de rasage entre les poses.

Je te parle de lexique; tu me dis que je manque de vocabulaire subtil, tacite, onomatopéen, mutiste. 
Glauque comme le sceau de sécurité autour de l'installation de la toilette.

Glauque comme nos veines de lagune dans la cotte de maille des sourires orthodontiques.

Glauque comme l'éternité interrompue par le gel à rasage.

Le vert bleuté de gommette à fixation et à affichage : le bleu verdi des cadavres

compostés.

C'est l'automne, et les chantiers de construction sont en fleurs fanées et en labour de fosses.

C'est l'automne, et les Bruins sont en vacances.

C'est l'automne, et tu me plantes un gril de calendrier dans le cœur.

C'est l'automne, et la poésie est inversement proportionnelle au temps qui nous reste.

C'est l'automne, et les enfants, les flots, nous éduquent.

C'est l'automne, et la première fumée des cheminées est

glauque comme les retailles de pelouse sur le plancher du garage.

C'est l'automne, et ma pâte à coiffer Axe a l'air du fard à ski de fond sur tes paupières.

C'est l'automne, et je voulais des enfants qui allaient me donner la main quand

j'arriverais, comme les trois feuilles éternellement vertes durant le givre et le Super Bowl de Cheerios.

C'est l'automne, et nous faisons de la drave sur le tronc de l'arbre généalogique qui a dû être abattu pour la préservation de l'horizon électrique.

C'est l'automne, et les feuilles éternelles sont glauques in the park, tapissant le ciel de plagiaire du pentagoniste.

Je plagie la Terre en photo, depuis la planète de tes yeux. 